\title{
Rare Iatrogenic Oronasal Communication after Superior Canine Odontectomy Attempt
}

\author{
Ciprian Roi ${ }^{1}$, Emilia Ianeș ${ }^{1}$, Diana Nica ${ }^{1}$, Alexandra Roi ${ }^{2, *}$, Laura Cristina Rusu ${ }^{2}$, \\ Ioana Badea ${ }^{3}$ and Mircea Riviș ${ }^{1}$
}

${ }^{\mathrm{I}}$ Department of Anaesthesiology and Oral Surgery, "Victor Babes" University of Medicine and Pharmacy Timisoara, 2Eftimie Murgu Square, 30004I Timisoara, Romania; ciprian.roi@umft.ro (C.R.); emilia.ianes@umft.ro (E.I.); nica.diana@umft.ro (D.N.); rivis.mircea@umft.ro (M.R.)

${ }^{2}$ Department of Oral Pathology, "Victor Babes" University of Medicine and Pharmacy Timisoara,

2Eftimie Murgu Square, 30004I Timisoara, Romania; laura.rusu@umft.ro (L.C.R)

3 PhD Student, "Victor Babes" University of Medicine and Pharmacy Timisoara, 2 Eftimie Murgu Square, 30004I Timisoara, Romania; badeaioanadaniela@yahoo.com (I.B)

*Correspondence: alexandra.moga@umft.ro; Tel.: +40-726080oo

Submitted: Is December 2020; Accepted: 22 January 2021; Published: 26 January 202I

\begin{abstract}
I) Background: Oronasal communication is described in the scientific literature as a common complication that occurs after a cleft palate surgery. In some cases, it can also be a consequence of oral surgery procedures; the main problem of this type of rare accident is related to the correct healing process and treatment option. (2) Case report: A patient with oronasal communication caused by an unsuccessful attempt of a superior canine odontectomy presented at the Emergency Department of the Oral and Maxillofacial Surgery Hospital, Timișoara. The case management is described from the first consult to one-year follow-up. We consider that this pathology was optimally treated surgically, and the results are more than satisfactory, taking into consideration the high rates of recurrence. (3) Conclusion: This case report can be a useful to a general dentist who is trying to decide whether to perform the canine odontectomy or refer to a specialist surgeon due to the accidents and complications of this procedure.
\end{abstract}

Keywords: oronasal communication; iatrogenic; oral surgery; local flaps; canine odontectomy

How to cite: Roi, C.; Ianeş, E.; Nica, D.; Roi, A.; Rusu, L.C.; Badea, I.; Riviș, M. Rare Iatrogenic Oronasal Communication after Superior Canine Odontectomy Attempt. Timisoara Med. 2020, 2020(2), 4; doi:I0.35995/tmj20200204.

\section{Introduction}

Oronasal communication is a rare accident that can occur during oral surgery procedures as exodontia of superior incisors and canines, odontectomies of impacted tooth in premaxilla area, apical tooth resection, tumour extirpation or cystectomy [I]. It can also appear as a complication due to trauma, gunshot injuries, syphilitic gumma, Caldwell-Luc procedure, radiation therapy, orthognathic surgery, cleft palate repair, and cocaine consumption $[2-5]$. 
This abnormal communication is a pathological continuity tract between the oral and nasal cavity with important functional disorders and repercussions, such as nasal regurgitation of the fluid and food, hypernasality of speech that may be accompanied by compensatory articulation disorders, whose gravity depend on the size of the communication [6]. Other symptoms include nasal obstruction, frequent crusting, accumulation of food particles within the communication, halitosis, leakage of nasal secretions into the mouth and Eustachian tube dysfunction. Sometimes, these symptoms can cause psychological problems $[5,7]$.

The surgical closure of the oronasal communication can involve palatal transposition flaps, palatal rotation, tongue flaps, buccal advancement flaps, and nasolabial flaps [8]. Suture dehiscence, post-operative infection, avascular necrosis and closure under tension remain major causes of recurrence. Among all complications of surgical closure, recurrence remains to be most common with rate of $25-37 \%$ with a challenging problem to surgeons. Primary closure in single layer often leads to failure and persists as a major concern to the operative surgeon [9].

\section{Case Report}

The patient, a 48-years-old male, presented at the Emergency Department of Oral and Maxillofacial Surgery Hospital, Timişoara, one week after an unsuccessful attempt of right superior canine odontectomy, performed in a dental office. Previously, on the X-ray, the tooth I.3 was identified in intraosseous impaction. After plexus anaesthesia, the odontectomy of the canine was intended and the approach was mixed: buccal flap and palatal flap. Even if the osteotomy of the maxillary bone was performed, the canine could not be discovered and the decision of the clinician was to suture the flaps, temporize the surgical procedure and refer the patient further to a department of maxillofacial surgery.

The main complaints of the patient at presentation were persistent pain, halitosis, masticatory perturbances and minor nasal fluid regurgitation. The general medical history of the patient had nothing relevant to show. We performed a loco-regional examination of the patient and the written informed consent of regarding the surgical treatment was obtained.

The intraoral examination revealed the presence of the erupted maxillary teeth I.7, I.2, I.I, 2.I, 2.4, 2.5, and 2.7 , a fact that was noted in the medical chart. In the anterior buccal side, a sutured flap was present. On the palatal side, the entire flap located in the premaxilla in relation to teeth I.2, I.I and 2.I was necrotic and sutured, surrounded by a hyperaemic mucosa (Figure I). An oro-nasal communication was present, with and a positive air circulation through the palatal flap (Figure 2).

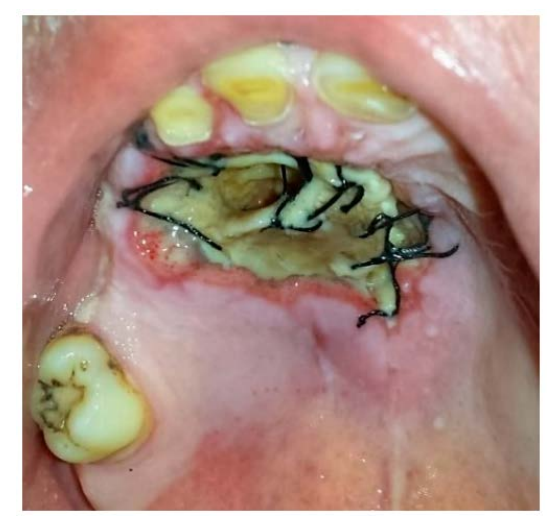

Figure 1. Necrosis of the palatal flap. The initial oral examination of the patient. 


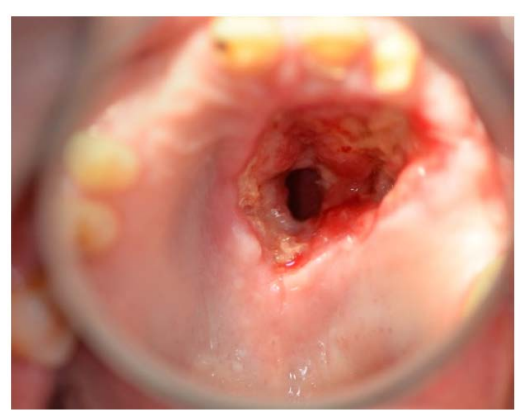

Figure 2. Oronasal communication aspect after removing the necrotic debris.

The first therapeutic step was the cleaning of the wound with antiseptic solutions-Povidone-Iodine Io\% and Chlorhexidine Digluconate $0.2 \%$, followed by suture removal and curettage of the necrotic tissues. The palatal bone could be identified without periosteum. In depth, the nasal cavity was exposed, and nasal secretions were leaking to the oral cavity. For the certain diagnostic to be confirmed, a full maxillary Cone Beam Computer Tomography (CBCT) exploration was performed (Figures 3 and 4 ).

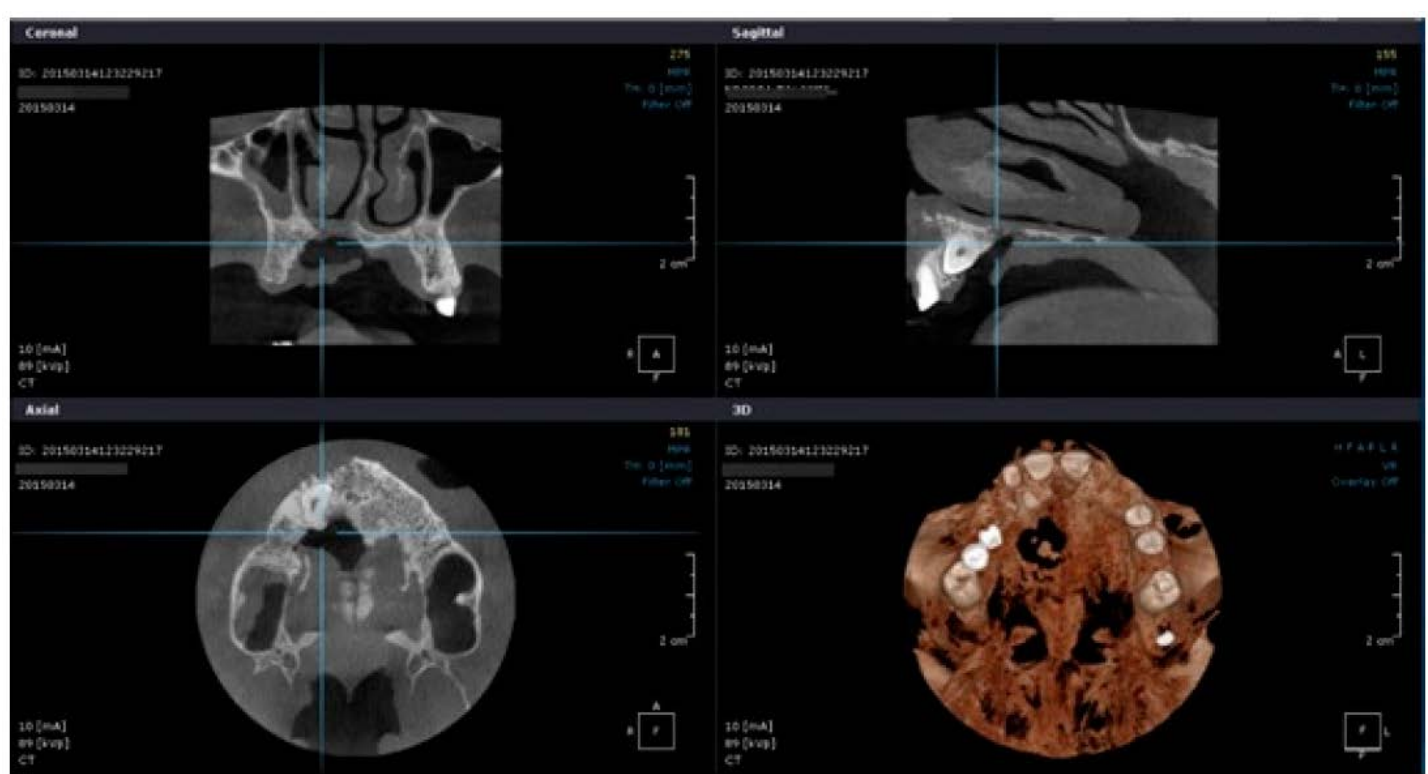

Figure 3. Oronasal communication: Cone Beam Computer Tomography (CBCT) aspect.

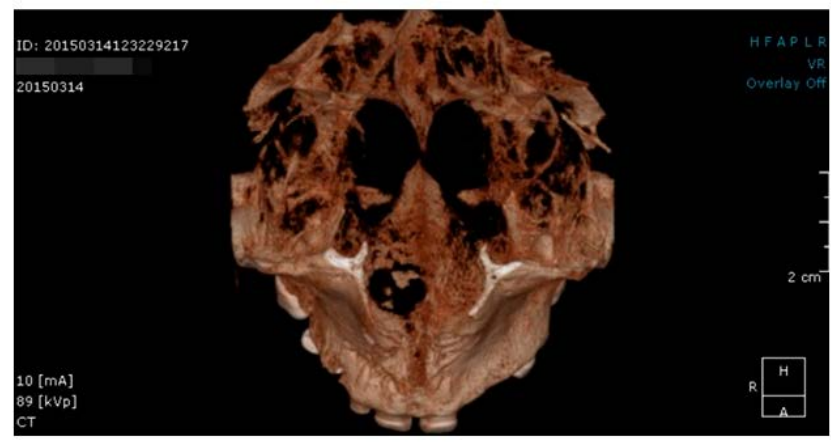

Figure 4. Oronasal communication: $\mathrm{CBCT}$ virtual $3 \mathrm{D}$ reconstruction. 
The patient was hospitalized and the medication was administrated intravenously: antibiotics, Clindamycin $600 \mathrm{mg} 3$ times/day; pain killers, Metamizole $\mathrm{g} / 2 \mathrm{~mL} 3$ times/day; and nonsteroidal anti-inflammatory drugs (NSAID), Ketoprofen Ioo $\mathrm{mg} / 2 \mathrm{~mL}$ twice daily for 7 days. The patient rinsed his mouth with mouthwash containing 0.2\% Chlorhexidine Digluconate twice a day. Cleaning the wound with Povidone-iodine Io\% was performed twice a day. Nasal saline spray was used in order to prevent maxillary sinusitis.

A dental impression was taken for the fabrication of a proper palatal obturator (Figure 5 ). The improvement of the symptoms was present and wound healing started to develop. The patient was discharged after io days and twice a week was recalled for a strict local hygiene.

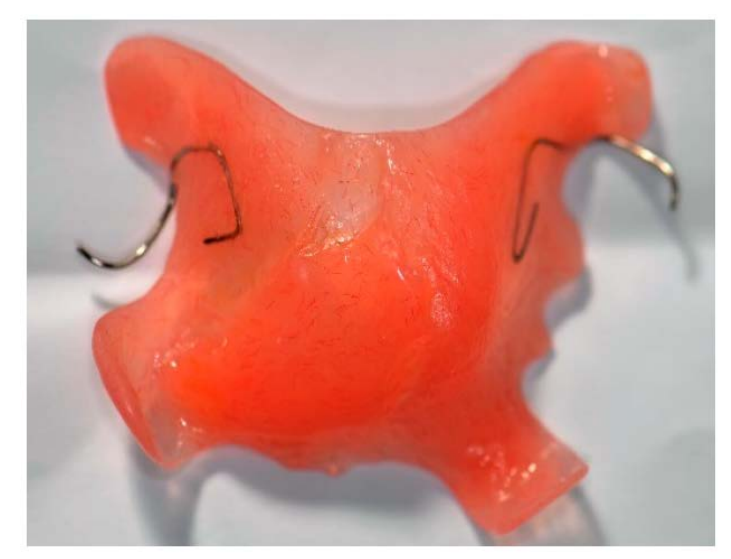

Figure 5. Palatal obturator.

Homecare in this case is very important, the patient being instructed to maintain the personalized palatal obturator during feeding and drinking and to perform proper oral hygiene. During the periodic check-ups, the diameter of the oronasal communication decreased and the epithelization was active. After 5 months, the entire part of the premaxillary fibromucosa was epithelized (where the necrotic area was initially present) and the palatal bone was covered by fixed oral mucosa. The oronasal communication had a round shape, with $5^{-6} \mathrm{~mm}$ in diameter, clean margins covered by normal oral mucosa, without bleeding or hyperaemic areas, with nasal secretions that were leaking through the fistula. Anterior from the oronasal communication tract, the cusp of the impacted canine was revealed (Figure 6).

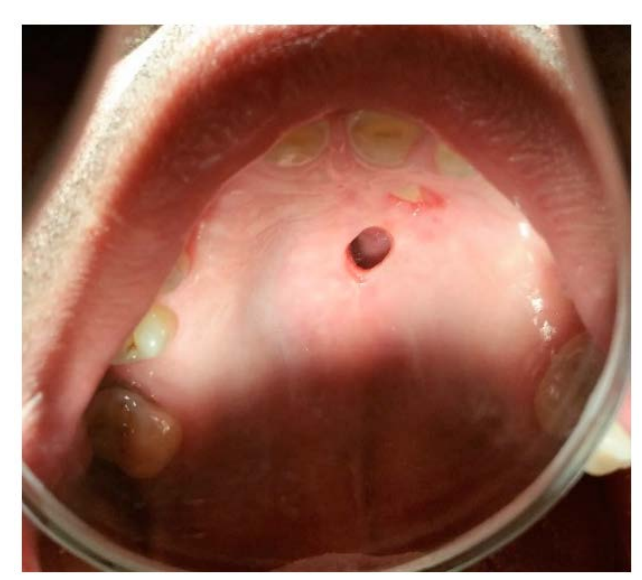

Figure 6. Oronasal communication after 5 months. The cusp of the impacted canine is revealed. 


\section{Surgical Steps}

The surgical approach in this case was considered after the primary healing of the nearby tissue and the remission of the inflammatory symptoms. After 3 months, the patient was hospitalized for surgical closure of the oronasal communication. First, the odontectomy of the canine was performed through a palatal approach (Figure 7). Bilateral greater palatine and maxillary incisive nerve anaesthesia were performed. The local anaesthetic substances used were Articaine $4 \%$ with I:200,ooo Epinephrine.

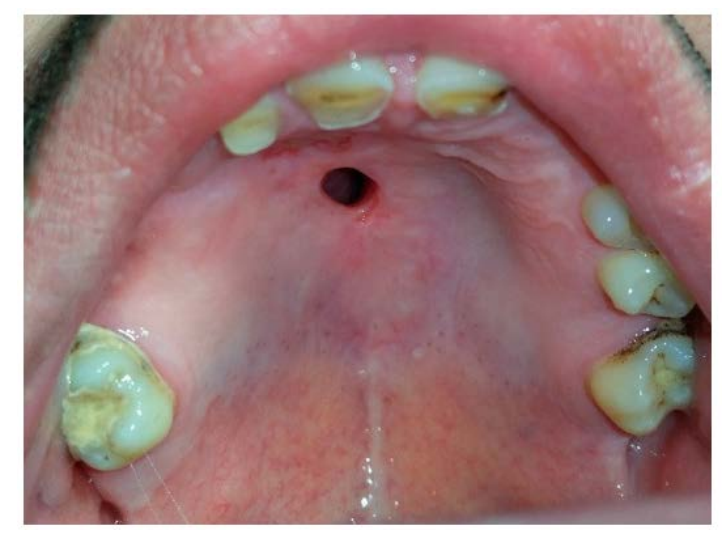

Figure 7. Oronasal communication after 6 months. The canine was extracted.

With a No. I5 scalpel blade, an incision around the communication was made, about $1.5 \mathrm{~cm}$ away from the margins. An oral mucosa collar was made and detached very close to the nasal side of the communication and sutured with 4-o Vicryl suture. This leads to inward movement of palatal mucosa into the nasal cavity, offering a barrier between the oral nasal cavity and providing a primary closure between these two anatomic cavities. Inward movement of mucosa exposes the submucosa to the oral cavity and provides a favourable raw and healthy surface for receiving a second layer closure. The second pedicle flap was based on the greater palatine artery. A full-thickness incision was performed, and a contralateral pedicled palatal rotation axial flap based on greater palatine artery was used as a second layer of closure, above the first palatal submucosal layer. The flap was sutured tension free with 3 -o silk, with alternation of interrupted suture and vertical mattress. The suture was removed after 7 days (Figure 8).

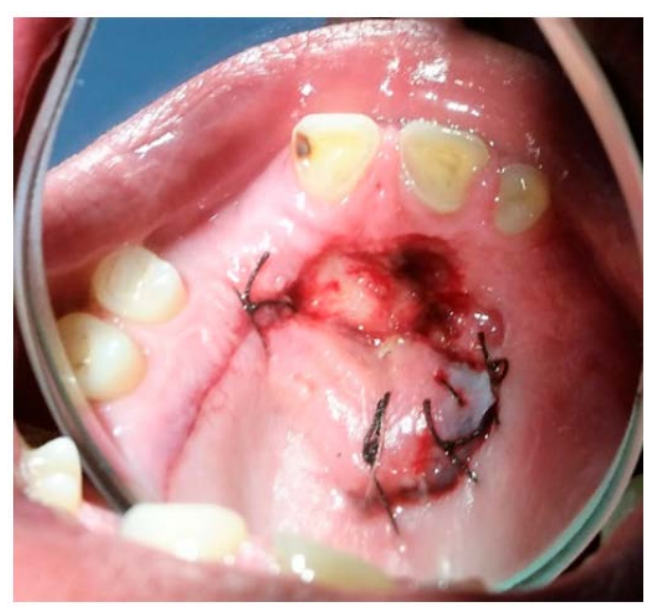

Figure 8. Local aspect at $24 \mathrm{~h}$ after surgery. 
Intravenous therapy was continued: antibiotics, Amoxicillin with clavulanic acid $875 \mathrm{mg} / \mathrm{r} 25 \mathrm{mg}$, twice daily; pain killers, Metamizole $\mathrm{g} / 2 \mathrm{~mL}, 3$ times/day; and nonsteroidal anti-inflammatory drugs (NSAID), Ketoprofen $100 \mathrm{mg} / 2 \mathrm{~mL}$, twice daily for 7 days. The patient rinsed his mouth using a mouthwash with $0.2 \%$ Chlorhexidine twice daily. The palatal obturator was used further on for preventing flap dehiscence (Figures 9 and $\mathrm{ro}$ ).

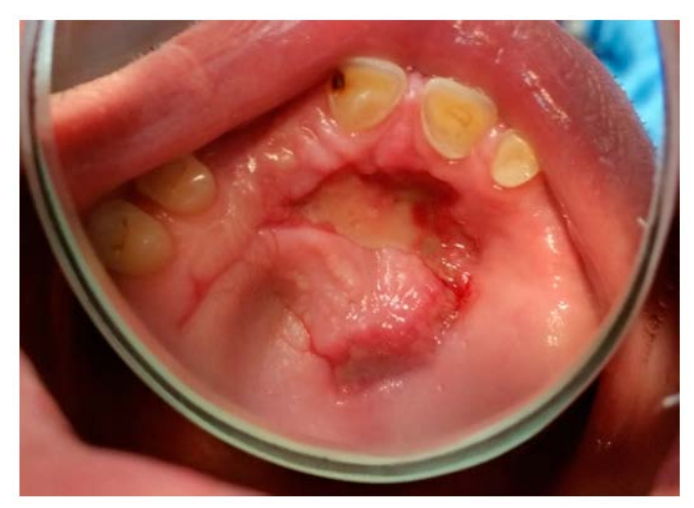

Figure 9. Local aspect at to days after surgery.

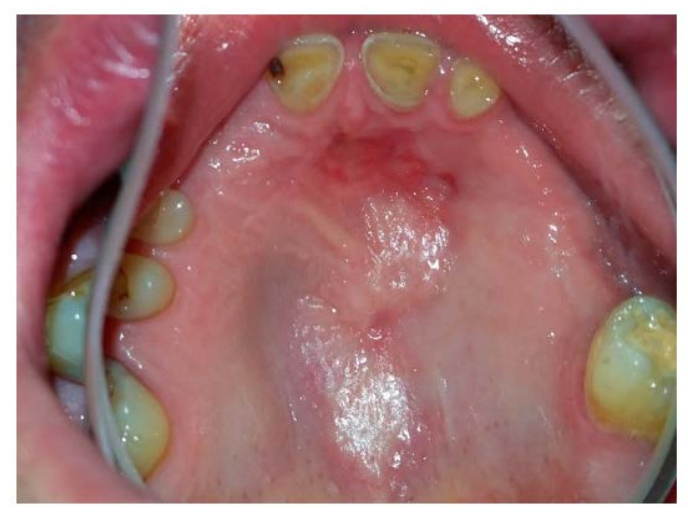

Figure 10. Local aspect at one month after surgery.

After one year, the patient was re-examined and no signs of an oronasal fistula were discovered. The OPG $\mathrm{X}$-ray showed that the maxillary bone was completely healed (Figure II).

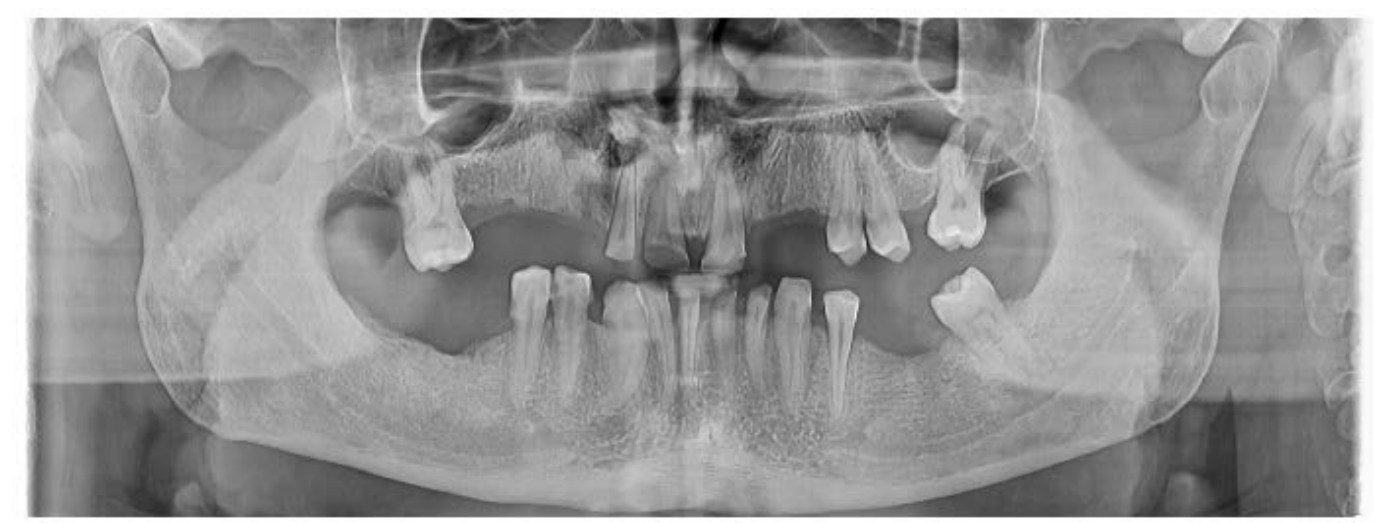

Figure 11. X-ray aspect at one year after the surgery. 


\section{Discussion}

The management of oronasal communication is challenging to perform due to the anatomy of the involved area: poor vascularization, presence of the nasal secretions in the oral cavity, high chance of the flap dehiscence, size of the oronasal communication and risk of infection. Although, the spontaneous closure of the communication is never possible based on the anatomical considerations [Io]. Closure techniques described in the scientific literature varied due to the dimensions of the communication, from one or two layers mucoperiosteal flap, to buccal advancement flaps, tongue flap, palatal transposition flaps, palatal rotation, nasolabial flaps or free vascularized tissue $[8, \mathrm{II}-\mathrm{I} 3]$.

The transfer of free vascularized tissue is mainly used for the large oronasal communication. It seems to be the most reliable and logical solution for medium- to large-sized fistulas. The most used flaps are from the anterolateral thigh, the brachial, the parascapular and the radial forearm [13].

The main advantages of the double-layered surgical closure of oronasal communication are represented by the perfect coverage of the defect with strength and vascularity improvements, reduced recurrence rate. This intramucosal purse string suture technique uses local tissue as an alternative option for double-layered closure. This option is characterized by low donor site morbidity and increased patient comfort [9].

Newer procedures like the use of bone morphogenic protein, acellular dermal matrices, human amniotic membrane, and distraction osteogenesis have a role to play in the management of oronasal communication [14].

In our case, the right decision was to wait for the healing of the necrotic maxillary bone and oral mucosa in order to gain sufficient local tissue resources and avoid the postoperative complications. It is important to mention that our patient had no medical problems, and the healing potential was exploited.

The main functional disorders caused by the oronasal communication must disappear after the surgery. These disorders are escaping air from nose to the oral cavity, presence of nasal secretions in oral cavity, integrity lack of the nasal floor and hard palate, hyper nasality of voice, affected resonance and nasal regurgitation of fluids $[6,10,15]$. In the present case report, after the final surgical step none of the mentioned functional disorders persisted, obtaining complete local healing with no further complications. A complete documentation of the case and an assessment of the potential intra-operatory accidents and long-term complications is mandatory in order to perform this type of surgical intervention.

\section{Conclusions}

Oronasal communication is a rare pathologic entity with major functional disorders for patient life. This abnormal communication can occur due to multiple etiologic factors. In our case, a simple canine odontectomy was transformed in a failure. The management of the case involved the extraction of the impacted upper canine and surgical sealing the oral cavity of the nasal cavity. The final result of the case was very satisfactory and all the functions of the oral cavity were restored.

Author Contributions: Conceptualization, C.R., and E.I.; Investigation, D.N.; Writing-Original Draft Preparation, M.R., L.C.R.; Writing-Review \& Editing, M.R. and A.R.; Visualization, I.B.; Supervision, C.R. and A.R.

Funding: This research received no external funding.

Conflicts of Interest: The authors declare no conflicts of interest. 


\section{References}

I. Kaur, P.; Kaur, J. Oronasal Fistula and Complete Edentulism: What to Do? Dent. J. 2014, 2, I42-I54. [CrossRef]

2. Eppley, B.; Sclaroff, A. Oronasal fistula secondary to maxillary augmentation. Int. J. Oral Surg. 1984, 13, 535-538. [CrossRef]

3. Emory, R.; Clay, R. Fistula formation and repair after palatal closure: An institutional perspective. Plast. Reconstr. Surg. 1997, 99, 1535-1538. [CrossRef] [PubMed]

4. Jeyaraj, P.E. A Study on Dorsal Pedicled Tongue Flap Closure of Palatal Fistulae and Oronasal Communications. Ann. Maxillofac. Surg. 2017, 7, 180-187. [CrossRef] [PubMed]

5. Hsu, Y.T.; Hao, S.P. Repair of oronasal fistula with silicone button in patients with head and neck cancer. Eur. Arch. Otorbinolaryngol. 2015, 272, I477-I48I. [CrossRef] [PubMed]

6. Jeyaraj, C.P. Techniques to Improve Reliability and Predictability of the Dorsal Pedicled Tongue Flap in Closure of Palatal Defects and Oronasal Fistulae. J. Maxillofac. Oral Surg. 2018, 17, 175-18I. [CrossRef] [PubMed]

7. Jeffery, S.L.; Boorman, J.G.; Dive, D.C. Use of cartilage grafts for closure of cleft palate fistulae. Br. J. Plast. Surg. 2000, 53, 55I-554. [CrossRef] [PubMed]

8. Kumar, V.V.N.P.; Kashyap, S.; Haidry, N.; Singh, M.; Rangaswamy, S.; Ranganatha, N. Buccal Fat Pad for Closure of Oroantral and Oronasal Communication: A Study of I6 Cases. Int. J. Oral Care Res. 2018, 6, $27-31$.

9. Singh, V.; Agarwal, S.K.; Jajodia, N.; Garg, A. Purse String Suture Closure: A Useful Double-Layer Technique for Closure of an Oronasal Communication. J. Maxillofac. Oral Surg. 2019, 18, 317-319. [CrossRef] [PubMed]

Io. Mohammed, V.A.; Farah, N. Repair of iatrogenic oronasal fistula after periapical surgery. Univ. Res. J. Dent. 2012, 2, 83-86.

II. Manimaran, K.; Sureshkannan, P.; Kannan, R. Oro Nasal Fistula Closure by Tongue Flap. JIADS 2011, 2, $60-62$.

I2. Fagan, J. Buccal Fat Pad Flap, Atlas of Otolaryngology, Head \& Neck Operative Surgery. Available online: https://vula.uct.ac.za/access/content/group/basfbrbd-be95-48e5-8rbe-586fbaeba29d/Buccal\%2ofat\% 2opad\%2oflap-I.pdf(Accessed on I2.II.2O20).

I3. Colletti, G.; Allevi, F.; Valassina, D.; Bertossi, D.; Biglioli, F. Repair of Cocaine-Related oronasal fistula with forearm radial free flap. J. Craniofac. Surg. 2013, 24, 1734-1738. [CrossRef] [PubMed]

I4. Sahoo, N.K.; Desai, A.P.; Roy, I.D.; Kulkarni, V. Oro-nasal communication. J. Craniofac. Surg. 2016, 27, e529-e533. [CrossRef] [PubMed]

15. Henningson, G.; Isberg, A. Oronasal fistulas and speech production. In Multidisciplinary Management of Cleft Lip and Palate, Ist ed.; WB Saunders: Philadelphia, PA, USA, I990; pp. 787-79I.

$\bigodot_{202 \mathrm{I}}$ Copyright by the author. Licensed as an open access article using a CC BY 4.0 license.

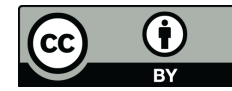

